\title{
LSST \\ AGN Science Collaboration Roadmap
}

Prepared by the LSST AGN Science Collaboration, with support from the LSST Corporation

Version 1.0

January 24, 2018 


\section{Contributing Authors}

Ohad Shemmer, ${ }^{1}$ Gordon Richards, ${ }^{2}$ Niel Brandt, ${ }^{3}$ Roberto Assef, ${ }^{4}$ Yan-Fei Jiang, ${ }^{5}$ Aaron Barth, ${ }^{6}$ Franz Bauer, ${ }^{7}$ Matt Jarvis,${ }^{8}$ Mark Lacy, ${ }^{9}$ Andy Lawrence, ${ }^{10}$ Paulina Lira, ${ }^{11}$ Chelsea MacLeod, ${ }^{12}$ Carole Mundell, ${ }^{13}$ Jeff Newman, ${ }^{14}$ Christina Peters ${ }^{15}$ et al.

${ }^{1}$ University of North Texas

${ }^{2}$ Drexel University

${ }^{3}$ The Pennsylvania State University

${ }^{4}$ Universidad Diego Portales

${ }^{5}$ University of California, Santa Barbara

${ }^{6}$ University of California, Irvine

${ }^{7}$ Pontificia Universidad Católica de Chile

${ }^{8}$ University of Oxford

${ }^{9}$ National Radio Astronomy Observatory

${ }^{10}$ University of Edinburgh

${ }^{11}$ Universidad de Chile

${ }^{12}$ Harvard-Smithsonian Center for Astrophysics

${ }^{13}$ University of Bath

${ }^{14}$ University of Pittsburgh

${ }^{15}$ University of Toronto 


\section{Contents}

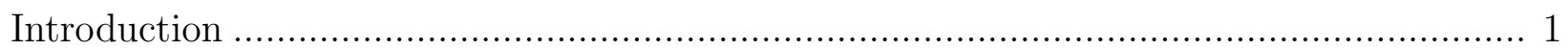

1 AGN Selection, Classification, and Characterization ….............................................. 2

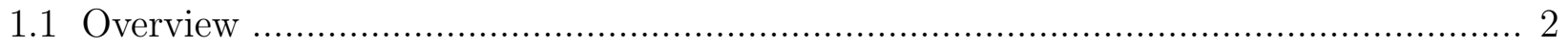

1.2 Unobscured Quasars/AGN Selection Methods ...................................................... 2

1.2.1 Major AGN SC Tasks Ranked by Decreasing Priority ……................................... 4

1.3 Transient, Obscured, and Low-Luminosity AGN Selection Methods ........................... 5

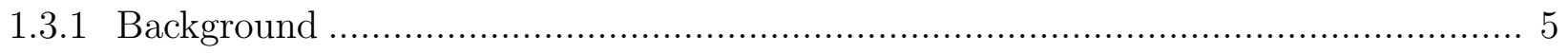

1.3.2 Major AGN SC Tasks Ranked by Decreasing Priority ……................................... 6

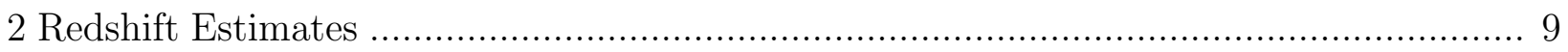

2.1 Overview ……

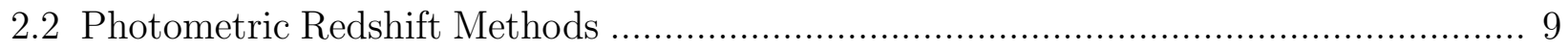

2.2.1 Major AGN SC Tasks Ranked by Decreasing Priority …….................................. 10

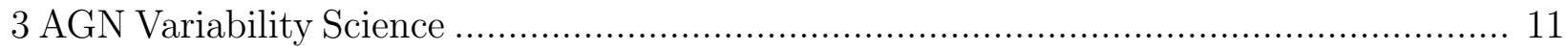

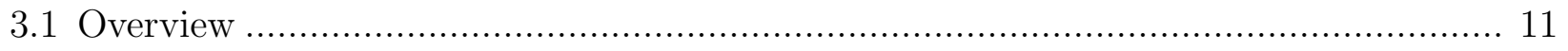

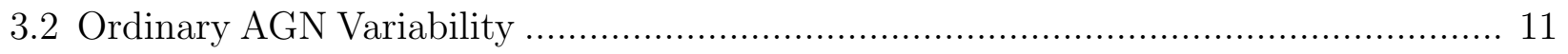

3.2.1 Major AGN SC Tasks Ranked by Decreasing Priority …….................................. 12

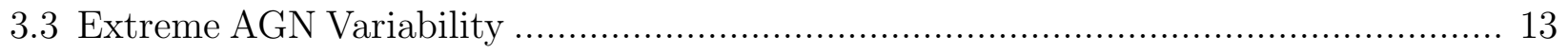

3.3.1 Major AGN SC Tasks Ranked by Decreasing Priority …...................................... 14

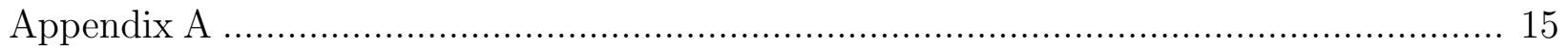

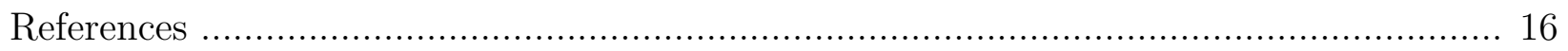




\section{Introduction}

\section{Ohad Shemmer}

The LSST AGN Science Collaboration (SC) held a meeting on January 3, 2017 in Grapevine, TX. The main goal was to start the development of a comprehensive Roadmap that will guide the SC activities during the pre- and post-commissioning phase of the LSST survey. The Roadmap presented below is intended to serve as an internal AGN SC, living document that builds on past and current LSST-related AGN studies, including the AGN chapters in the LSST Science Book (Abell et al. 2009), the LSST Observing Strategy White Paper (Marshall et al. 2017), and the various contributions that can be linked to from the LSST AGN SC website. ${ }^{1}$ Its purpose is to describe and prioritize critical AGN science goals, many of which were identified at the meeting, along with tentative timelines to achieving these goals.

Three dedicated Working Groups (WGs), composed of AGN SC members, have been formed during the Grapevine meeting in order to work on specific projects described by the Roadmap. These WGs are focused on: 1) AGN Selection, Classification, and Characterization, 2) Redshift Estimates, and 3) AGN Variability Science. Each WG and its tasks/projects are described in detail in each of the following chapters, which appear in the order that paves the way to AGN investigations during and after LSST operations. The first chapter is devoted to optimizing AGN selection in order to construct the largest possible census. The second is focused on developing methods for improving the crucial redshift information. The last chapter involves precursor AGN variability studies in order to optimize AGN selection and prepare for the arrival of millions of AGN light curves.

Each chapter begins with a general overview, followed by sections dedicated to a particular topic or topics. The structure of each section includes a short background, followed by a ranked list of major tasks in order of decreasing priority; several sections end with a paragraph detailing additional, lower-priority tasks. Each major task can be viewed as an abstract for a particular stand-alone project. Interested SC members are encouraged to work on one or more of these projects and to seek funding support for their performance. Additional, more specific questions regarding these projects should be forwarded to the chapters'

leading authors. The task lists are not considered to be final, and AGN SC members are encouraged to suggest additional tasks/project along with their suggested priorities

\footnotetext{
${ }^{1}$ https://agn.science.lsst.org/
} 


\title{
Chapter 1: AGN Selection, Classification, and Characterization
}

\author{
Gordon Richards, Niel Brandt, Franz Bauer, et al.
}

\subsection{Overview}

For all AGN science with LSST, the first goal is seemingly straightforward: identify efficient and complete ways to pinpoint the location of actively accreting supermassive black holes (SMBHs) on the sky, whether through short-lived transient events or longer-lived fueling. However, optimal methods for doing so are not expected to be generic for all types of AGN, which can be split broadly into four major classes: unobscured quasars/AGN, obscured quasars/AGN, low-luminosity AGN (LLAGN), and transient SMBH fueling events. In the following sections of this chapter, we highlight the challenges LSST will face for each of these classes of objects and we identify the most important goals for the AGN SC in coming years for addressing those challenges.

Construction of LSST's AGN census will build upon a considerable volume of past work. Color-selection has been the gold standard for identification of unobscured quasars since their discovery (e.g., Koo \& Kron 1982; Warren et al. 1991; Richards et al. 2002). Identification of quasars by their variable nature (e.g., Bonoli et al. 1979; Trevese et al. 1989; Butler \& Bloom 2011) and lack of proper motion (e.g., Sandage \& Luyten 1967; Kron \& Chiu 1981) are also not new. Even the idea of performing a multi-faceted quasar selection (e.g., colors, variability, and proper motion) is not new (e.g., Koo et al. 1986). However, at the same time, the quality, quantity, and type of data that LSST will provide will allow more complete AGN selection and thus these approaches must be considered from a "new again" perspective.

Historically, the selection of AGN whether via color, variability, or proper motion has relied on "cuts" in the parameter space in question. This process works quite well in that it produces samples that are sufficiently efficient that spectroscopic follow-up is cost effective. However, such methods are not statistically optimal and only in recent years have we seen the implementation of "machine learning" methods (e.g., Richards et al. 2004; Bovy et al. 2011). This field is growing rapidly and LSST science stands to benefit considerably from considering all of the latest research in statistics and computer science (e.g., Feigelson \& Babu 2012). Below, we highlight the most pressing tasks that are needed to accomplish the goal of optimal identification of classical type-1 AGN (i.e., those dominated by their central engines). In a subsequent Section we consider the other three AGN classes.

\subsection{Unobscured Quasars/AGN Selection Methods}

Colors/Flux Ratios: Color-selection by itself is certainly the most mature of the avenues for identifying AGN (unobscured or otherwise). Any application of modern statistical techniques to color data will be a major first step in the process. It is unlikely that any final method(s) adopted for LSST AGN selection will be completely color based, thus it will be important to extend such efforts to multi-parameter selection methods using the information from the following methods.

Astrometry and (Lack of) Proper Motion: As some AGN and stars have very similar colors, the fact that AGN do not have proper motions (while Galactic stars do) has long been used as a discriminant. LSST's use of astrometric data will be no different in that regard. Where LSST will be unique is in its ability to take advantage of differential chromatic 
refraction (DCR) of AGN (Kaczmarczik et al. 2009). In short, the DCR procedure makes use of the astrometric offset of an emission-line object from that expected (in the astrometric solution) for a power-law source. Here we consider DCR's use for selection; see Chapter 2 for its use with redshift estimation.

Variability: As quasars vary in their brightness much more than the average star and in a much different way than the typical variable star, variability will be a cornerstone of AGN classification for LSST. However, variability by itself is unlikely to be a panacea. Even for luminous quasars, it has been shown that variability combined with colors works better for selection than variability alone (Peters et al. 2015). Moreover, even though lower-luminosity AGN are expected to have the most variable nuclei, increasing contamination from the host galaxy will compromise variability-selection methods if insufficient care is taken.

Multiwavelength Data: The fourth way that LSST will identify unobscured AGN is by combining with multiwavelength data. This can be considered more generally as "combination with data from other facilities" as some data (e.g., Euclid) may also be in the optical. In nearly all cases we will have tiered multiwavelength data to contend with: shallow over a large area to deep over a small area. For unobscured AGN, multiwavelength data will enhance our ability to identify high-redshift quasars and can be used to modify the AGN probabilities for objects on the border; this process will be most useful within the context of a probabilistic redshift distribution (see Chapter 2). Objects detected in the X-ray or IR with sufficiently high predicted luminosity will have increased AGN probability. Objects that are bright in the UV will have significantly decreased AGN probability (for high photometric redshifts), while objects that are radio bright will have increased probability. Currently, the greatest depth of the full sky is 2MASS in the near-IR, WISE in the mid-IR, ROSAT (and soon eROSITA) in the X-ray, and NVSS in the radio (soon to be replaced by EMU/Wodan). Somewhat deeper, but with less coverage we have UKIDSS and VHS in the near-IR; SpIES, SSDF, SERVS, SDWFS, and SWIRE in the mid-IR; the XMM-Newton Slew Survey and the Chandra Source Catalog in the X-ray; and GALEX in the UV. The key deep fields will be located within the deep-drilling fields (DDFs), e.g., X-SERVS which will become important in training general selection in the DDFs (Chen et al. 2018, submitted).

LSST will optimize the identification of unobscured AGN using the following relevant parameters:

- color

- variability

- astrometry (DCR and proper motion)

- brightness

- Galactic coordinates

- morphology

- probability of belonging to another class

- multiwavelength and multi-facility matching

Ideally, all of these will be used simultaneously. Here we only have considered the inputs to the selection algorithms in generic terms; however, in Appendix A, we specifically identify the database entries that represent those attributes in the context of the current LSST "Data Model". 


\subsubsection{Major AGN SC Tasks Ranked by Decreasing Priority}

1. Establish public training/test sets that AGN SC members can use as a benchmark to test different selection algorithms (i.e., a "data challenge" sample). This could include (or be completely based upon) simulated data. It must include color, astrometry, timedomain, and multiwavelength information needed to test all of the processes described above. One example is HSC optical imaging in the XMM-LSS and COSMOS fields.

2. Generate realistic catalog-level simulated data. Such simulations would need to cover as much "area" and as many epochs as possible in all six LSST bands in a format that mimics the yearly data releases. As much physics and empirical correlations as possible must be included, such as: luminosity-dependence of emission features, magnitudecolor correlations, variability physics, astrometric errors, DCR, proper motion, nuclear vs. host galaxy luminosity correlation (and its relationship to morphology), star-galaxy separation, lensing probability, broad absorption lines and dust reddening (intrinsic, host galaxy, and intervening). In other words, the simulations must account for known quasar spectral diversity and its effects on LSST photometry.

3. Determine what "color" means in the LSST context. Application of the color-based facet of the selection algorithm(s) requires that the AGN SC first determine how we will compute the colors (and/or flux ratios) — given lack of simultaneous data in multiple bandpasses. Alternatively, we could explore methods that do not compute a single "color" for each object but rather operate on the full multi-band light curves. Nevertheless, it will be important for display purposes to establish what is meant by the colors of each object.

4. Explore different machine learning techniques (e.g., Random Forests, Support Vector Machines, Gaussian Mixture Models, Deep Learning, etc.), including both parametric and non-parametric approaches. The algorithms in standard machine learning libraries generally lack the ability to 1) output probabilities, 2) take Bayesian priors (e.g., brightness and Galactic latitude), 3) handle errors, and 4) handle missing data. The AGN SC must interact with the Informatics SC in this regard to adapt and test different machine learning algorithms in order to determine the optimal approaches.

5. Continue to test the effects of different cadence schemes on AGN selection (and AGN physics; Marshall et al. 2017). While the default plan is for uniform cadence, there are a number of proposals for cadences that deviate from uniform. It is not inherently obvious whether AGN science would benefit or suffer from each of these algorithms. Therefore, each needs to be tested with explicitly-defined metrics. A working hypothesis is that AGN may be best served by a cadence that is uniform in $\log \Delta t$ (e.g., with spacing akin to a Golomb ruler) rather than in time. The AGN SC should therefore develop its own cadence proposal, generate an $\mathrm{OpSim}^{2}$ that uses it and evaluates it (along with OpSims that represent other cadences) within the Metric Analysis Framework $(\mathrm{MAF})^{3}$ (with the metric itself also to-be-determined).

\footnotetext{
${ }^{2}$ https://www.lsst.org/scientists/simulations/opsim

${ }^{3}$ https://www.lsst.org/scientists/simulations/maf
} 
6. Work with (and simulate) asynchronous light curves for variability selection. LSST time domain analysis will be utilizing information more efficiently if data from multiple bands are combined to create a "single" light curve. The AGN SC must determine how best to merge multi-band light curves (e.g., kriging), accounting for (or determining) colors, brightness-color dependence, time delays, non-simultaneity, intergalactic absorption, etc. Moreover, traditional variability analysis requires a parametric fit to the data. The possibility of performing non-parametric analysis (i.e., simply using the full, raw light curves) should be investigated and if a parametric fit is needed, determine the best form(s) (e.g., CARMA, DRW, Slepian wavelet variance, etc.; see also Mahabal et al. 2017). Furthermore, given that AGN variability is nuclear, the AGN $\mathrm{SC}$ should investigate the possibility of performing such analysis in the context of difference imaging (building on work from other SCs like those working on supernovae). Many of these issues can be investigated with existing (and ongoing) data sets such as Stripe 82, HSC, DES, Pan-STARRS, Catalina, Kepler, etc.

7. Establish "truth tables" in the DDFs for multiwavelength selection. The LSST project needs to begin to tabulate spectroscopic (and multiwavelength) information in the currently-defined DDFs and establish a mechanism for storing and updating said information. Multiwavelength photometry must be incorporated, using Tractor-based forced photometry at the fiducial optical position (from data most similar to LSST). Alternatively (or in addition), implement true SED-based band merging (e.g., Budavari \& Szalay 2008). Establish a 'wish list' for additional spectroscopy in the DDFs prior to the start of survey operations.

8. Develop luminosity function and clustering algorithms that work with probabilistic AGN candidates and apply these algorithms to the simulated data. Write papers based on the results that illustrate the size of the uncertainties that LSST data will produce given the model assumptions.

9. Establish a plan for working with intermediate products in the first year of the survey (e.g., reference images and prior classifications).

Additional important tasks that the SC should attend to on a short timescale include (i) appointment of expert "ambassadors" for each major source of multiwavelength data, (ii) continue ongoing discussions with other LSST SCs and groups (e.g., improve star-galaxy separation techniques in conjunction with the Galaxies SC, work with DM group to ensure good AGN-host deblending, folding multiwavelength data back into the LSST Data Model, whether or not to use photo- $z$ 's as feedback to produce rest-frame quantities), and (iii) interpretation of LSST Data Model outputs and how they relate to the key parameters mentioned above as well as relevant parameters that were used for the analysis of SDSS data (e.g., proper motion information based on USNO and GAIA data, morphology characterization, variability information, and novel DCR parameters that LSST will produce).

\subsection{Transient, Obscured, and Low-Luminosity AGN Selection Methods}

\subsubsection{Background}


A key goal for the LSST AGN SC is to optimize AGN selection to enable the largest possible census. While "traditional" selection/classification/characterization methods are expected to drive the selection of bright AGN in the yearly data releases, there are three motivations for investigating additional "non-traditional" methods. For instance, the nominal angular resolution of LSST should allow separation of galaxy and nuclear components, enabling us to extend the selection and characterization of AGN nuclei well below the traditional "quasar limit" (i.e., LLAGN) where unresolved host-galaxy contamination had previously become dominant. At the extreme limit of LLAGN are another class, namely obscured AGN, where there is little or no optical continuum emission from the AGN along our line of sight.

For obscured AGN and LLAGN, the optical data from LSST may not be sufficient to identify the object as such. Some LLAGN may be identified as having nuclear variability, but for the faintest objects the errors on the variability will be insufficient for robust classification. As such, for these classes of objects we will be dependent upon multiwavelength SED fitting and X-ray or IR and/or radio luminosity and radio morphology to identify as an AGN what LSST sees as a galaxy. While we focus here on obscured AGN and LLAGN, many of the tasks listed below will also be related to identifying AGN that are unique in other bandpasses (e.g., blazars). In order to avoid repetition with respect to procedures required for the identification of unobscured AGN (e.g., creating a data challenge set, testing machine learning algorithms, establishing truth tables), these tasks are specifically geared towards identifying/classifying transient, obscured, and low-luminosity AGN.

Lastly, in order to capitalize on the unique time-domain capabilities of LSST, we should be prepared to assess the potential for "extreme" variability on a nightly basis in order to trigger immediate, rapid follow-up programs of extreme AGN events, for example, tidal disruption events (TDEs). This naturally feeds into the AGN variability WG (see Chapter 3 ).

\subsubsection{Major AGN SC Tasks Ranked by Decreasing Priority}

1. Test host and nuclear component fitting/decomposition, which is required for extracting nucleus photometry/colors.

2. Determine what special measurements are needed/desired to select LLAGN. The most general constraint should be the point-spread function (PSF) magnitude assessed from the difference image at the location of the galaxy nucleus at each epoch ( $\Delta m$ detection or upper limit).

3. For dwarf galaxies and irregulars, where a central nucleus may be ill or undefined, one needs to explore whether the variability of aperture photometry over the entire extent of the source, or whether relying on difference imaging, would allow for a more efficient selection. In other words, one needs to determine whether it is better to use the position of the variable source within the galaxy to eliminate contaminants or attempt to improve the photometry of the source.

4. Determine the utility of folding into the selection algorithm the host color and morphology, redshift/intrinsic properties, and multiwavelength properties. Test whether these 
parameters enhance detection rates and increase AGN probabilities, and investigate the potential for bias.

5. Use existing data and DDFs (e.g., HSC, X-SERVS, etc.) to determine over what range of parameter space can we successfully select LLAGN. As no real systematic studies of the optical variability of LLAGN have been made, we will be exploring new territory here. As such, need to prepare for the unknown and be as open as possible to a large parameter range.

6. There is a need to identify special measurements required for TDE selection. For example, a PSF magnitude from a galaxy nucleus to assess variability. Furthermore, there is a need to establish a definition for "strong" or "extreme" variability (e.g., on average, a factor of 5 or 30 increase in amplitude, respectively), and develop a framework for distinguishing between large increases and decreases in flux. Such variability criteria should be determined in a statistical manner based on distributions of variability amplitudes as functions of other properties (e.g., luminosity). in this context, it is important to consider more exotic possibilities such as sources exhibiting "strong" variability coincident with no observed galaxy or AGN, in consultation with the Transients and Variable Stars SC.

7. There is a need to determine the response timescales required for follow-up observations of TDEs, blazars, microlensing events, and changing-look quasars (CLQs) for AGN selection purposes. This also requires development of trigger programs and determining how these will be implemented. Additionally, we should determine whether the AGN $\mathrm{SC}$ needs a dedicated event broker for this purpose as opposed to collaborating with a general broker for AGN variability science (see Chapter 3).

8. A 'typical' TDE should have no spectroscopic signs of an AGN prior to the outburst. This is obviously impossible to test after the outburst but one should develop some photometric criterion, e.g., the surface brightness distribution at/near the nucleus before the outburst, that will enable figuring out if there was a strong, blue, nuclear point source before the outburst. An alternative way to test that, which is beyond the scope of this Roadmap, would be through spectroscopy at a very late time (many years in the rest frame) after the outburst. These TDEs can be compared to events where a prior AGN is identified in order to establish how such events differ individually and collectively from their typical counterparts.

9. In order to maximize TDE selection, we need to explore TDE decay laws that differ from the canonical $t^{-5 / 3}$ dependence after the initial outburst, as recent suggestions imply that other decay laws (e.g., an exponential decay) may be possible.

10. Determine whether there is a need for increased cadence photometry and/or timedependent color/SED information for TDEs and CLQs.

11. There should be a plan in place for follow-up spectroscopy and multiwavelength imaging of TDEs, CLQs, and other extreme AGN identified by LSST. 
12. LSST will undoubtedly find new types of AGN. We should start thinking about what we can expect and how would we select such sources.

Additional important tasks that the SC should attend to on a short timescale include (i) recruiting "ambassadors" who are experts in galaxy morphology and in transients from the Transients and Variable Stars SC, and (ii) identifying critical parameters that are not provided by the DM group. 


\section{Chapter 2: Redshift Estimates}

Roberto Assef, Franz Bauer, Niel Brandt, Matt Jarvis, Mark Lacy, Jeff Newman, Christina Peters, Gordon Richards, et al.

\subsection{Overview}

Redshift estimates for the vast majority of LSST AGN will have to rely on photometric redshifts, or photo- $z$, techniques. There is a long history of broad-band photometric redshift estimation techniques, although these are most commonly applied to galaxies without nuclear activity. AGN are not only much less common than inactive galaxies, but also their photometric redshift estimates can be much less accurate. It will be important for the AGN SC to optimize and characterize the photo- $z$ method(s) to be used, particularly in terms of their accuracy and biases in light of the specific science goals pursued. In general, photometric redshift techniques can be divided into two broad families: those that rely on SED template fitting, and those that rely on empirical correlations. We briefly discuss each of them in the following, as well as additional improvements that could be implemented.

\subsection{Photometric Redshift Methods}

SED Template Fitting: SED template fitting is one of the most popular techniques for photometric redshift estimates of galaxies (e.g., Bolzonella et al. 2000; Benitez 2000). In their simplest version, SED template fitting methods operate by taking a number of different, pre-selected SED templates, computing their broad-band colors as a function of redshift, and selecting the template and redshift that best match the observed photometry. Emission lines typically do not have large enough equivalent widths to affect broad-band colors within the photometric accuracy, so broad features such as the Balmer and Lyman break in the optical, as well as rest-frame $1.6 \mu \mathrm{m}$ peak in the near-IR, provide the majority of the information for photometric redshift estimates. For obscured AGN or LLAGN, where the host galaxy dominates the optical SED, template methods provide the most accurate redshift estimates. Moreover, Assef et al. (2010) note that SED template fitting techniques can provide accurate host galaxy-AGN decompositions, even when suffering from highly inaccurate redshift estimates. Hence, such SED modeling techniques should be considered for science goals requiring SED decomposition, even if the redshift estimates are obtained through a different method.

Empirical Methods: While template methods have been proven to work well for galaxies without nuclear activity and obscured AGN, the lack of such broad features in the spectra of luminous quasars (except for the Lyman break at high redshift) makes these methods highly inaccurate (see, e.g., Rowan-Robinson et al. 2008; Salvato et al. 2009; Assef et al. 2010). To estimate the redshifts of luminous quasars, empirical methods (e.g., Weinstein et al. 2004; Brodwin et al. 2006; Richards et al. 2009) provide much better redshift accuracy and hence have been strongly preferred. These methods rely on a training set with known redshifts from which they construct empirical relations between the inputs, typically (but not limited to) broad-band photometry, and the redshift itself. Because of this, these methods can incorporate in a natural manner many other observational properties than simply the observed broad-band fluxes, which is a complicated task for SED template fitting meth-

ods. Empirical methods can be based on a number of different approaches, such as neural 
networks, machine learning algorithms and Bayesian kernel density estimates. As long as a training sample that is representative of the LSST AGN sample can be constructed, or at least one that is well characterized, these methods should be much preferable over SED template fitting for luminous quasars.

\subsubsection{Major AGN SC Tasks Ranked by Decreasing Priority}

1. Determine all the possible sources of information (colors, fluxes, variability, DCR, multiwavelength data, etc.) that can be used by photometric redshift algorithms.

2. Determine how to use existing data (Stripe 82, DES, etc.) to test the scheme to characterize DCR. Interface with the DM group to determine what DCR parameter will be produced.

3. Identify the actual data set(s) needed to create an initial training set. Make this training set public so that it can be used as a benchmark to test different algorithms.

4. Identify the empirical method(s) to be used for the more luminous sources (see also Chapter 1), and identify SED fitting methods that would be useful for LLAGN and obscured AGN.

5. Consider hybrid classification/regression. As empirical photo- $z$ methods will use the same information that would be used for classification, it should be possible to join both processes and perform the identification and photo- $z$ estimation simultaneously. Need to explore generation of this information as an LSST Level 2 (rather than Level 3) product.

6. Compare the effectiveness of all the photo- $z$ methods and identify the optimal solution(s). Compare the results to sources that have independent galaxy photo- $z$ estimates.

7. Determine the expected photo- $z$ accuracy, considering subsamples of different characteristics (e.g., LLAGN, obscured AGN, and luminous AGN).

8. Develop a MAF that assesses how a given OpSim (e.g., with a rolling cadence and/or high airmass observations) changes the accuracy of the chosen quasar photo- $z$ method(s).

9. Construct a spectroscopic follow-up plan for the DDFs that would allow the training set to be modified after the first year of the survey.

10. Determine what additional data (i.e., data external to LSST) can be added for the photo- $z$ estimations and on which timescales. This can include data from, e.g., Euclid, WFIRST, or eROSITA.

11. Modify photo- $z$ algorithms to produce probability distribution functions (PDFs) rather than single values. Study how one should store the PDF information in an efficient manner. Develop quasar luminosity function and clustering algorithms that work with PDF photo- $z$ data. 


\section{Chapter 3: AGN Variability Science}

Yan-Fei Jiang, Carole Mundell, Ohad Shemmer, Chelsea L. MacLeod, Aaron Barth, Paulina Lira, Christina Peters, Andy Lawrence, et al.

\subsection{Overview}

Aside from playing a leading role in the selection of LSST AGN, AGN variability can provide invaluable information about the immediate surroundings of actively accreting SMBHs, including both the accretion disk and broad emission line region (BELR). This information can, ultimately, provide estimates of SMBH mass and accretion power. Different AGN variability timescales, from days, months, to years, in the rest frame, can be directly connected to the dynamical, thermal, and inflow timescales of the accretion disks. Finding statistically robust models to describe the AGN light curves is crucial for these studies. The unprecedented number of multi-band light curves that LSST will provide, covering a wide range of timescales, will enable testing different models of AGN variability that can break the degeneracies between the potential controlling parameters and improve our understanding of the underlying physical processes. AGN variability science is likely to be impacted the most due to changes in the nominal LSST cadence. Therefore, a significant fraction of the AGN SC work should be directed toward assessing the impact of potential LSST observing strategies and identifying potentially harmful cadences.

\subsection{Ordinary AGN Variability}

Ordinary AGN variability concerns light fluctuations originating in the accretion disk or BELR of an actively accreting SMBH. The characteristic timescales observed in such variations provide a direct link between observations and theoretical models of the accretion disk and BELR. In the simplest case, the shortest observable timescale (light-crossing time) places an upper limit on the size of the variable emission region. Then there are the dynamical and sound-crossing timescales (for a summary, see Lawrence et al. 2016). In accretion-disk theory, the thermal and viscous timescales correspond to the heat dissipations and radial inflow rates, respectively, and have often been related to the observed timescales in quasar light curves (Lyubarskii 1997; Kelly et al. 2009, 2011; Kasliwal et al. 2017). Microlensing measurements of half-light radii of accretion disks always find that the measured value is larger than the predicted value by a factor of 3-4 (Morgan et al. 2010; Mosquera et al. 2013; Chartas et al. 2016). These results inspired alternative models of accretion disks (Dexter \& Agol 2011). Because the effective temperature of accretion disks decreases with increasing distance to the SMBH, emission in different wavelength bands is dominated by different locations on the disk.

Measuring the time lags between the variabilities of different continuum bands is another way to constrain the properties of accretion disks (Edelson et al. 2015; Jiang et al. 2017). The color variability of AGN has also been shown to exhibit a tight "bluer when brighter" trend (Schmidt et al. 2012). This is another way to distinguish different accretion disk models (Ruan et al. 2014; Zhu et al. 2016). Light curves with daily cadences for a large sample of AGN covering wide ranges of wavelength bands, that can be provided by LSST, will place a tight constraint on the radial dependence of the effective temperature in the accretion disks. The longer lags, on typical timescales of weeks to months, in the rest frame, between the 
continuum and the BELR lines will be important for probing the sizes of the BELRs and testing the dependence of the BELR on AGN luminosity.

Ordinary AGN variability can be described statistically with various processes ranging from a damped random walk (DRW) to quasi-periodic oscillation (QPO). The analysis of AGN light curves, particularly when the sampling pattern is irregular with few data points, typically relies on a structure function (SF) which is a robust way to estimate variability timescales that can be compared to a DRW model (Kozlowski 2016). The SF can also be modeled by a broken power-law power spectrum distribution (PSD) with multiple break time scales (Kasliwal et al. 2017), or a mixed Ornstein-Uhlenbeck (OU) process (Kelly et al. 2011). Variability on short timescales is sometimes better described by DRW+QPO, or the 2nd order Continuous time AutoRegressive Moving Average (CARMA) process. The latter is described by second-order stochastic linear differential equations, while DRW is, intrinsically, the first-order CARMA process. It has been shown recently that the secondorder CARMA process is a better descriptor for some Kepler AGN (Kasliwal et al. 2015) and for $\sim 30 \%$ of Optical Gravitational Lensing Experiment (OGLE) AGN (Zinn et al. 2017).

Circumbinary accretion disks have been theorized to occur around binary SMBHs, and the time-variable mass accretion rate can potentially produce periodic flux variability in the object's light curve (Artymowicz \& Lubow 1996). Furthermore, the interaction between the binary SMBH and the gaseous accretion disk can play a major role in the binary orbital evolution and help drive the binary toward its eventual merger (e.g., Rafikov 2013). Since ordinary AGN variability is aperiodic, identifying compelling cases of periodically variable quasars would be an important step toward demonstrating the existence of binary SMBHs and studying their properties.

Searches for periodically variable quasars have uncovered an increasing number of candidates using data from the Catalina Real-Time Transient Survey (CRTS) and from PanSTARRS (Graham et al. 2015a,b; Liu et al. 2015; Zheng et al. 2016). Typical examples from CRTS have candidate periods of $\sim 2-4 \mathrm{yr}$, corresponding to estimated binary separations of order $\sim 10^{-2}$ pc (Graham et al. 2015b). Some caution is warranted in periodicity searches, however, since DRW or other stochastic behavior can easily produce spurious quasi-periodicities over a short duration, and some false positives are likely to be found when searching large quasar samples. A necessary step is to carry out realistic Monte Carlo simulations to test any possible variability signal against the null hypothesis of aperiodic behavior, in order to assess the probability of a false positive periodicity signal (Vaughan et al. 2016). Once a candidate periodic quasar is identified, further monitoring is needed over longer timescales to test whether the past periodicity predicts future behavior. Any confirmed periodic quasars will be important targets for extensive follow-up to determine their physical properties, and could provide potential candidates for gravitational-wave studies. LSST will excel at producing quasar light curves with high signal-to-noise, long duration, and frequent sampling, which will provide the best database for identifying and verifying possible cases of periodic variability.

\subsubsection{Major AGN SC Tasks Ranked by Decreasing Priority}

1. There is a need to simulate the AGN light curves for a variety of SMBH masses, Eddington ratios, and at different redshifts (e.g., Zinn et al. 2017). The simulations 
should be done using different cadences to see how the light curve variability property can be recovered. The simulation(s) can rely on a sample of existing AGN to test the methods, choose the wavelength bands, and evaluate the use of non-simultaneous observations in different wavelength bands.

2. Both short and long timescale variabilities from a large sample of simulated LSST AGN light curves should be used to perform photometric reverberation mapping (PRM) to detect continuum-continuum and BELR line-continuum lags. These lags, particularly in the DDFs, can be used to constrain the size of the accretion disk and BELR for a large sample $\left(\approx 10^{4}-10^{5}\right.$ sources) of SMBH masses and Eddington ratios (e.g., Chelouche et al. 2014; Jiang et al. 2017).

3. Simulations should be done to test the effects of candidate cadences on potential continuum-continuum and BELR line-continuum lags. In particular, for quasars at redshifts with significant line flux at the photometric band, simulations need to be done to see how to separate the effects of BELR lines on the continuum-continuum lags. The first steps in this direction were taken by Chelouche et al. (2014) who explored the feasibility of PRM, given the nominal LSST survey parameters, and its sensitivity to basic sampling patterns. The main LSST survey is expected to deliver BELR line-continuum time delays in $\approx 10^{5}-10^{6}$ sources, although the measurement uncertainties will likely be large even when grouped in various parts of parameter space.

4. In the DDFs, where the sampling is expected to be an order of magnitude denser with respect to the main survey, PRM measurement uncertainties are expected to be more acceptable. In these fields, one should: 1) assess the ability to successfully (in terms of both quantity and quality) recover line-continuum time delays given different candidate cadences, especially for potential rolling cadences, and to identify particularly harmful cadences, and 2) plan precursor spectroscopic observations of sources for which PRM will likely yield reliable line-continuum time delays that will lead to SMBH mass estimates given accurate line-width measurements.

5. In preparation for LSST, simulations need to be performed to assess how well periodic signals can be detected based on realistic AGN light curves. These simulations can add periodic signals to mock AGN light curves (for example, generated by a DRW model) to test the ability to recover periodicity as a function of signal-to-noise, sampling, monitoring duration, variability amplitude, and period. Tests can also be carried out to assess the false positive rate using intrinsically aperiodic light curves.

6. Results from pre- and post-commissioning variability science should be fed back into AGN selection in general.

\subsection{Extreme AGN Variability}

Variability is a key characteristic of AGN, capable of probing size scales that are otherwise not directly measurable, giving us SMBH masses, and setting a severe challenge to AGN theories; for example, the optical-UV variability observed is too fast for simple accretion disk theories, and are puzzlingly simultaneous. These long standing issues have 
been re-awakened by a number of recent discoveries and trends, for example, TDEs, microlensed outbursts, blazars, flaring AGN, extreme variables and CLQs, and the realization that decadal timescales may be crucial to understanding AGN physics. LSST is perfectly designed to make progress in these areas, but needs to be accompanied by well organized spectroscopic observations. Some of the requirement is for contemporaneous observations, but we also need precursor observations, and links to historic samples.

\subsubsection{Major AGN SC Tasks in Decreasing Priority}

1. The AGN SC should develop a strategy for triggering (mostly rapid) follow-up observations (photometric and spectroscopic) of different AGN variability events including, e.g., transient AGN, microlensing, and CLQs, not just for classification purposes. In the LSST era, this cannot rely on an individual object, hand-crafted approach, but rather on a "factory" approach. Therefore, we need to design and implement predictive algorithms that bring such extreme events (e.g., a microlensing event approaching a cusp, a blazar flare, or the peak of a CLQ outburst) to our attention, and interface with event brokers from other science collaborations.

2. Develop a strategy for precursor massive spectroscopic monitoring on the longest timescales. Many spectra with high signal-to-noise ratios over timescales of years-todecades, exploiting several legacy surveys (e.g., Stripe 82) are required for identifying peculiar AGN or extreme AGN variability.

3. Investigate cadence effects on extreme AGN variability science given the likely rarity of these events and the different timescales of each.

4. There is a need to develop model light curves for various types of extreme AGN variability and pre-simulate a full range of predicted behaviors to quantify what we will be able to infer from the alert stream per night and cumulatively.

5. There is a need to identify sufficiently deep, wide-area, multi-band and multi-temporal blind data for developing and testing AGN SC event brokers. These brokers should assume response times ranging from days to weeks and distinguish between follow-up requiring small (i.e., $4 \mathrm{~m}$ ) telescopes and large (i.e., $8 \mathrm{~m}$ ) telescopes. These should also consider three different approaches to deal with the alerts: (i) issue an alert and allow others to respond, (ii) establish a global network of partners/facilities who would follow-up on the alerts, and (iii) propose for significant portions of target-of-opportunity time on various facilities, perhaps jointly with other SCs.

6. There is a need to develop careful host-subtraction techniques that would impact, e.g., TDE discovery.

7. Results from from extreme variability science should be fed back into AGN selection in general. 
Appendix A

Coming soon... 


\section{References}

1. LSST Science Collaboration, Abell, P.A., Allison, J., Anderson, S.F., Andrew, J.R., Angel, J.R.P., Armus, L., Arnett, D., Asztalos, S.J., Axelrod, T.S., Bailey, S., Ballantyne, D.R., Bankert, J.R., Barkhouse, W.A., Barr, J.D., Barrientos, L.F., Barth, A.J., Bartlett, J.G., Becker, A.C., Becla, J., Beers, T.C., Bernstein, J.P., Biswas, R., Blanton, M.R., Bloom, J.S., Bochanski, J.J., Boeshaar, P., Borne, K.D., Bradac, M., Brandt, W.N., Bridge, C.R., Brown, M.E., Brunner, R.J., Bullock, J.S., Burgasser, A.J., Burge, J.H., Burke, D.L., Cargile, P.A., Chandrasekharan, S., Chartas, G., Chesley, S.R., Chu, Y.-H., Cinabro, D., Claire, M.W., Claver, C.F., Clowe, D., Connolly, A.J., Cook, K.H., Cooke, J., Cooray, A., Covey, K.R., Culliton, C.S., de Jong, R., de Vries, W.H., Debattista, V.P., Delgado, F., Dell'Antonio, I.P., Dhital, S., Di Stefano, R., Dickinson, M., Dilday, B., Djorgovski, S.G., Dobler, G., Donalek, C., Dubois-Felsmann, G., Durech, J., Eliasdottir, A., Eracleous, M., Eyer, L., Falco, E.E., Fan, X., Fassnacht, C.D., Ferguson, H.C., Fernandez, Y.R., Fields, B.D., Finkbeiner, D., Figueroa, E.E., Fox, D.B., Francke, H., Frank, J.S., Frieman, J., Fromenteau, S., Furqan, M., Galaz, G., Gal-Yam, A., Garnavich, P., Gawiser, E., Geary, J., Gee, P., Gibson, R.R., Gilmore, K., Grace, E.A., Green, R.F., Gressler, W.J., Grillmair, C.J., Habib, S., Haggerty, J.S., Hamuy, M., Harris, A.W., Hawley, S.L., Heavens, A.F., Hebb, L., Henry, T.J., Hileman, E., Hilton, E.J., Hoadley, K., Holberg, J.B., Holman, M.J., Howell, S.B., Infante, L., Ivezic, Z., Jacoby, S.H., Jain, B., R, Jedicke, Jee, M.J., Garrett Jernigan, J., Jha, S.W., Johnston, K.V., Jones, R.L., Juric, M., Kaasalainen, M., Styliani, Kafka, Kahn, S.M., Kaib, N.A., Kalirai, J., Kantor, J., Kasliwal, M.M., Keeton, C.R., Kessler, R., Knezevic, Z., Kowalski, A., Krabbendam, V.L., Krughoff, K.S., Kulkarni, S., Kuhlman, S., Lacy, M., Lepine, S., Liang, M., Lien, A., Lira, P., Long, K.S., Lorenz, S., Lotz, J.M., Lupton, R.H., Lutz, J., Macri, L.M., Mahabal, A.A., Mandelbaum, R., Marshall, P., May, M., McGehee, P.M., Meadows, B.T., Meert, A., Milani, A., Miller, C.J., Miller, M., Mills, D., Minniti, D., Monet, D., Mukadam, A.S., Nakar, E., Neill, D.R., Newman, J.A., Nikolaev, S., Nordby, M., O'Connor, P., Oguri, M., Oliver, J., Olivier, S.S., Olsen, J.K., Olsen, K., Olszewski, E.W., Oluseyi, H., Padilla, N.D., Parker, A., Pepper, J., Peterson, J.R., Petry, C., Pinto, P.A., Pizagno, J.L., Popescu, B., Prsa, A., Radcka, V., Raddick, M.J., Rasmussen, A., Rau, A., Rho, J., Rhoads, J.E., Richards, G.T., Ridgway, S.T., Robertson, B.E., Roskar, R., Saha, A., Sarajedini, A., Scannapieco, E., Schalk, T., Schindler, R., Schmidt, S., Schmidt, S., Schneider, D.P., Schumacher, G., Scranton, R., Sebag, J., Seppala, L.G., Shemmer, O., Simon, J.D., Sivertz, M., Smith, H.A., Allyn Smith, J., Smith, N., Spitz, A.H., Stanford, A., Stassun, K.G., Strader, J., Strauss, M.A., Stubbs, C.W., Sweeney, D.W., Szalay, A., Szkody, P., Takada, M., Thorman, P., Trilling, D.E., Trimble, V., Tyson, A., Van Berg, R., Vanden Berk, D., VanderPlas, J., Verde, L., Vrsnak, B., Walkowicz, L.M., Wandelt, B.D., Wang, S., Wang, Y., Warner, M., Wechsler, R.H., West, A.A., Wiecha, O., Williams, B.F., Willman, B., Wittman, D., Wolff, S.C., Wood-Vasey, W.M., Wozniak, P., Young, P., Zentner, A., and Zhan, H. 2009, 'LSST Science Book, Version 2.0', ArXiv e-prints, arXiv:0912.0201

2. Artymowicz, P. and Lubow, S.H. 1996, 'Mass Flow through Gaps in Circumbi- 
nary Disks', The Astrophysical Journal, 467, L77

3. Assef, R.J., Kochanek, C.S., Brodwin, M., Cool, R., Forman, W., Gonzalez, A.H., Hickox, R.C., Jones, C., Le Floc'h, E., Moustakas, J., Murray, S.S., and Stern, D. 2010, 'Low-Resolution Spectral Templates for Active Galactic Nuclei and Galaxies from $\mathbf{0 . 0 3}$ to $\mathbf{3 0} \mu \mathrm{m}$ ', The Astrophysical Journal, 713, 970-985

4. Benítez, N. 2000, 'Bayesian Photometric Redshift Estimation', The Astrophysical Journal, 536, 571-583

5. Bolzonella, M., Miralles, J.-M., and Pelló, R. 2000, 'Photometric redshifts based on standard SED fitting procedures', Astronomy and Astrophysics, 363, 476-492

6. Bonoli, F., Braccesi, A., Federici, L., Zitelli, V., and Formiggini, L. 1979, 'A study of the optical variability of the quasistellar objects in the $13^{\mathrm{h}}+36^{\circ}$ field. I The blue magnitudes', Astronomy and Astrophysics Supplement Series, 35, 391-401

7. Bovy, J., Hennawi, J.F., Hogg, D.W., Myers, A.D., Kirkpatrick, J.A., Schlegel, D.J., Ross, N.P., Sheldon, E.S., McGreer, I.D., Schneider, D.P., and Weaver, B.A. 2011, 'Think Outside the Color Box: Probabilistic Target Selection and the SDSSXDQSO Quasar Targeting Catalog', The Astrophysical Journal, 729, 141

8. Brodwin, M., Brown, M.J.I., Ashby, M.L.N., Bian, C., Brand, K., Dey, A., Eisenhardt, P.R., Eisenstein, D.J., Gonzalez, A.H., Huang, J.-S., Jannuzi, B.T., Kochanek, C.S., McKenzie, E., Murray, S.S., Pahre, M.A., Smith, H.A., Soifer, B.T., Stanford, S.A., Stern, D., and Elston, R.J. 2006, 'Photometric Redshifts in the IRAC Shallow Survey', The Astrophysical Journal, 651, 791-803

9. Budavári, T. and Szalay, A.S. 2008, 'Probabilistic Cross-Identification of Astronomical Sources', The Astrophysical Journal, 679, 301-309

10. Butler, N.R. and Bloom, J.S. 2011, 'Optimal Time-series Selection of Quasars', The Astronomical Journal, 141, 93

11. Chartas, G., Rhea, C., Kochanek, C., Dai, X., Morgan, C., Blackburne, J., Chen, B., Mosquera, A., and MacLeod, C. 2016, 'Gravitational lensing size scales for quasars', Astronomische Nachrichten, 337, 356

12. Chelouche, D., Shemmer, O., Cotlier, G.I., Barth, A.J., and Rafter, S.E. 2014, 'On the Performance of Quasar Reverberation Mapping in the Era of Time-domain Photometric Surveys', The Astrophysical Journal, 785, 140

13. Dexter, J. and Agol, E. 2011, 'Quasar Accretion Disks are Strongly Inhomogeneous', The Astrophysical Journal, 727, L24

14. Edelson, R., Gelbord, J.M., Horne, K., McHardy, I.M., Peterson, B.M., Arévalo, P., Breeveld, A.A., De Rosa, G., Evans, P.A., Goad, M.R., Kriss, G.A., Brandt, W.N., Gehrels, N., Grupe, D., Kennea, J.A., Kochanek, C.S., Nousek, J.A., Papadakis, I., Siegel, M., Starkey, D., Uttley, P., Vaughan, S., Young, S., Barth, A.J., Bentz, M.C., 
Brewer, B.J., Crenshaw, D.M., Dalla Bontà, E., De Lorenzo-Cáceres, A., Denney, K.D., Dietrich, M., Ely, J., Fausnaugh, M.M., Grier, C.J., Hall, P.B., Kaastra, J., Kelly, B.C., Korista, K.T., Lira, P., Mathur, S., Netzer, H., Pancoast, A., Pei, L., Pogge, R.W., Schimoia, J.S., Treu, T., Vestergaard, M., Villforth, C., Yan, H., and $\mathrm{Zu}$, Y. 2015, 'Space Telescope and Optical Reverberation Mapping Project. II. Swift and HST Reverberation Mapping of the Accretion Disk of NGC 5548', The Astrophysical Journal, 806, 129

15. Feigelson, E.D. and Babu, G.J. 2012, 'Modern Statistical Methods for Astronomy', Modern Statistical Methods for Astronomy, by Eric D. Feigelson, G. Jogesh Babu, Cambridge, UK: Cambridge University Press, 2012

16. Flaugher, B. and Bebek, C. 2014, 'The Dark Energy Spectroscopic Instrument (DESI)', Ground-based and Airborne Instrumentation for Astronomy V, 9147, $91470 \mathrm{~S}$

17. Graham, M.J., Djorgovski, S.G., Stern, D., Drake, A.J., Mahabal, A.A., Donalek, C., Glikman, E., Larson, S., and Christensen, E. 2015a, 'A systematic search for close supermassive black hole binaries in the Catalina Real-time Transient Survey', Monthly Notices of the Royal Astronomical Society, 453, 1562-1576

18. Graham, M.J., Djorgovski, S.G., Stern, D., Glikman, E., Drake, A.J., Mahabal, A.A., Donalek, C., Larson, S., and Christensen, E. 2015b, 'A possible close supermassive black-hole binary in a quasar with optical periodicity', Nature, 518, 74-76

19. Jiang, Y.-F., Green, P.J., Greene, J.E., Morganson, E., Shen, Y., Pancoast, A., MacLeod, C.L., Anderson, S.F., Brandt, W.N., Grier, C.J., Rix, H.-W., Ruan, J.J., Protopapas, P., Scott, C., Burgett, W.S., Hodapp, K.W., Huber, M.E., Kaiser, N., Kudritzki, R.P., Magnier, E.A., Metcalfe, N., Tonry, J.T., Wainscoat, R.J., and Waters, C. 2017, 'Detection of Time Lags between Quasar Continuum Emission Bands Based On Pan-STARRS Light Curves', The Astrophysical Journal, 836, 186

20. Kaczmarczik, M.C., Richards, G.T., Mehta, S.S., and Schlegel, D.J. 2009, 'Astrometric Redshifts for Quasars', The Astronomical Journal, 138, 19-27

21. Kasliwal, V.P., Vogeley, M.S., and Richards, G.T. 2015, 'Are the variability properties of the Kepler AGN light curves consistent with a damped random walk?', Monthly Notices of the Royal Astronomical Society, 451, 4328-4345

22. Kasliwal, V.P., Vogeley, M.S., and Richards, G.T. 2017, 'Extracting information from AGN variability', Monthly Notices of the Royal Astronomical Society, 470, 3027-3048

23. Kelly, B.C., Bechtold, J., and Siemiginowska, A. 2009, 'Are the Variations in Quasar Optical Flux Driven by Thermal Fluctuations?', The Astrophysical Journal, 698, 895-910 
24. Kelly, B.C., Sobolewska, M., and Siemiginowska, A. 2011, 'A Stochastic Model for the Luminosity Fluctuations of Accreting Black Holes', The Astrophysical Journal, $\mathbf{7 3 0}, 52$

25. Koo, D.C. and Kron, R.G. 1982, 'QSO counts - A complete survey of stellar objects to B = 23', Astronomy and Astrophysics, 105, 107-119

26. Koo, D.C., Kron, R.G., and Cudworth, K.M. 1986, 'Quasars to B greater than 22.5 in selected area 57 - A catalog of multicolor photometry, variability, and astrometry', Publications of the Astronomical Society of the Pacific, 98, 285-306

27. Kozłowski, S. 2016, 'Revisiting Stochastic Variability of AGNs with Structure Functions', The Astrophysical Journal, 826, 118

28. Kron, R.G. and Chiu, L.-T.G. 1981, 'Stars with zero proper motion and the number of faint QSOs', Publications of the Astronomical Society of the Pacific, 93, $397-404$

29. Lawrence, A. 2016, 'Clues to the Structure of AGN Through Massive Variability Surveys', Astronomical Surveys and Big Data, 505, 107

30. Liu, T., Gezari, S., Heinis, S., Magnier, E.A., Burgett, W.S., Chambers, K., Flewelling, H., Huber, M., Hodapp, K.W., Kaiser, N., Kudritzki, R.-P., Tonry, J.L., Wainscoat, R.J., and Waters, C. 2015, 'A Periodically Varying Luminous Quasar at z = 2 from the Pan-STARRS1 Medium Deep Survey: A Candidate Supermassive Black Hole Binary in the Gravitational Wave-driven Regime', The Astrophysical Journal, 803, L16

31. Lyubarskii, Y.E. 1997, 'Flicker noise in accretion discs', Monthly Notices of the Royal Astronomical Society, 292, 679

32. Mahabal, A., Sheth, K., Gieseke, F., Pai, A., Djorgovski, S.G., Drake, A., Graham, M., and the CSS/CRTS/PTF Collaboration 2017, 'Deep-Learnt Classification of Light Curves', ArXiv e-prints, arXiv:1709.06257

33. Majewski, S.R., Munn, J.A., Kron, R.G., Bershady, M.A., Smetanka, J.J., and Koo, D.C. 1991, 'A proper motion and variability QSO survey to $\mathbf{B}=\mathbf{2 2 . 5}$ ', The Space Distribution of Quasars, 21, 55-65

34. LSST Science Collaboration, Marshall, P., Anguita, T., Bianco, F.B., Bellm, E.C., Brandt, N., Clarkson, W., Connolly, A., Gawiser, E., Ivezic, Z., Jones, L., Lochner, M., Lund, M.B., Mahabal, A., Nidever, D., Olsen, K., Ridgway, S., Rhodes, J., Shemmer, O., Trilling, D., Vivas, K., Walkowicz, L., Willman, B., Yoachim, P., Anderson, S., Antilogus, P., Angus, R., Arcavi, I., Awan, H., Biswas, R., Bell, K.J., Bennett, D., Britt, C., Buzasi, D., Casetti-Dinescu, D.I., Chomiuk, L., Claver, C., Cook, K., Davenport, J., Debattista, V., Digel, S., Doctor, Z., Firth, R.E., Foley, R., Fong, W.f., Galbany, L., Giampapa, M., Gizis, J.E., Graham, M.L., Grillmair, C., Gris, P., Haiman, Z., Hartigan, P., Hawley, S., Hlozek, R., Jha, S.W., Johns-Krull, C., Kanbur, 
S., Kalogera, V., Kashyap, V., Kasliwal, V., Kessler, R., Kim, A., Kurczynski, P., Lahav, O., Liu, M.C., Malz, A., Margutti, R., Matheson, T., McEwen, J.D., McGehee, P., Meibom, S., Meyers, J., Monet, D., Neilsen, E., Newman, J., O’Dowd, M., Peiris, H.V., Penny, M.T., Peters, C., Poleski, R., Ponder, K., Richards, G., Rho, J., Rubin, D., Schmidt, S., Schuhmann, R.L., Shporer, A., Slater, C., Smith, N., Soares-Santos, M., Stassun, K., Strader, J., Strauss, M., Street, R., Stubbs, C., Sullivan, M., Szkody, P., Trimble, V., Tyson, T., de Val-Borro, M., Valenti, S., Wagoner, R., Wood-Vasey, W.M., and Zauderer, B.A. 2017, 'Science-Driven Optimization of the LSST Observing Strategy', ArXiv e-prints, arXiv:1708.04058

35. Morgan, C.W., Kochanek, C.S., Morgan, N.D., and Falco, E.E. 2010, 'The Quasar Accretion Disk Size-Black Hole Mass Relation', The Astrophysical Journal, 712, 1129-1136

36. Mosquera, A.M., Kochanek, C.S., Chen, B., Dai, X., Blackburne, J.A., and Chartas, G. 2013, 'The Structure of the X-Ray and Optical Emitting Regions of the Lensed Quasar Q 2237+0305', The Astrophysical Journal, 769, 53

37. Peters, C.M., Richards, G.T., Myers, A.D., Strauss, M.A., Schmidt, K.B., Ivezić, Ž., Ross, N.P., MacLeod, C.L., and Riegel, R. 2015, 'Quasar Classification Using Color and Variability', The Astrophysical Journal, 811, 95

38. Rafikov, R.R. 2013, 'Structure and Evolution of Circumbinary Disks around Supermassive Black Hole Binaries', The Astrophysical Journal, 774, 144

39. Richards, G.T., Fan, X., Newberg, H.J., Strauss, M.A., Vanden Berk, D.E., Schneider, D.P., Yanny, B., Boucher, A., Burles, S., Frieman, J.A., Gunn, J.E., Hall, P.B., Ivezić, Ž., Kent, S., Loveday, J., Lupton, R.H., Rockosi, C.M., Schlegel, D.J., Stoughton, C., SubbaRao, M., and York, D.G. 2002, 'Spectroscopic Target Selection in the Sloan Digital Sky Survey: The Quasar Sample', The Astronomical Journal, 123, 2945-2975

40. Richards, G.T., Nichol, R.C., Gray, A.G., Brunner, R.J., Lupton, R.H., Vanden Berk, D.E., Chong, S.S., Weinstein, M.A., Schneider, D.P., Anderson, S.F., Munn, J.A., Harris, H.C., Strauss, M.A., Fan, X., Gunn, J.E., Ivezić, Ž., York, D.G., Brinkmann, J., and Moore, A.W. 2004, 'Efficient Photometric Selection of Quasars from the Sloan Digital Sky Survey: 100,000 $z<3$ Quasars from Data Release One', The Astrophysical Journal Supplement Series, 155, 257-269

41. Richards, G.T., Myers, A.D., Gray, A.G., Riegel, R.N., Nichol, R.C., Brunner, R.J., Szalay, A.S., Schneider, D.P., and Anderson, S.F. 2009, 'Efficient Photometric Selection of Quasars from the Sloan Digital Sky Survey. II. 1,000,000 Quasars from Data Release 6', The Astrophysical Journal Supplement Series, 180, 67-83

42. Rowan-Robinson, M., Babbedge, T., Oliver, S., Trichas, M., Berta, S., Lonsdale, C., Smith, G., Shupe, D., Surace, J., Arnouts, S., Ilbert, O., Le Févre, O., Afonso-Luis, A., Perez-Fournon, I., Hatziminaoglou, E., Polletta, M., Farrah, D., and Vaccari, M. 
2008, 'Photometric redshifts in the SWIRE Survey', Monthly Notices of the Royal Astronomical Society, 386, 697-714

43. Ruan, J.J., Anderson, S.F., Dexter, J., and Agol, E. 2014, 'Evidence for Large Temperature Fluctuations in Quasar Accretion Disks from Spectral Variability', The Astrophysical Journal, 783, 105

44. Salvato, M., Hasinger, G., Ilbert, O., Zamorani, G., Brusa, M., Scoville, N.Z., Rau, A., Capak, P., Arnouts, S., Aussel, H., Bolzonella, M., Buongiorno, A., Cappelluti, N., Caputi, K., Civano, F., Cook, R., Elvis, M., Gilli, R., Jahnke, K., Kartaltepe, J.S., Impey, C.D., Lamareille, F., Le Floc'h, E., Lilly, S., Mainieri, V., McCarthy, P., McCracken, H., Mignoli, M., Mobasher, B., Murayama, T., Sasaki, S., Sanders, D.B., Schiminovich, D., Shioya, Y., Shopbell, P., Silverman, J., Smolčić, V., Surace, J., Taniguchi, Y., Thompson, D., Trump, J.R., Urry, M., and Zamojski, M. 2009, 'Photometric Redshift and Classification for the XMM-COSMOS Sources', The Astrophysical Journal, 690, 1250-1263

45. Sandage, A. and Luyten, W.J. 1967, 'On the Nature of Faint Blue Objects in High Galactic Latitudes. I. Photometry, Proper Motions, and Spectra in PHL Field 1:36 $+6^{\circ}$ and Richter Field M3, II', The Astrophysical Journal, 148, 767

46. Schmidt, K.B., Rix, H.-W., Shields, J.C., Knecht, M., Hogg, D.W., Maoz, D., and Bovy, J. 2012, 'The Color Variability of Quasars', The Astrophysical Journal, $\mathbf{7 4 4}, 147$

47. Süveges, M., Fotopoulou, S., Coupon, J., Paltani, S., Eyer, L., and Rimoldini, L. 2017, 'Learn from every mistake! Hierarchical information combination in astronomy', IAU Symposium, 325, 39-45

48. Trevese, D., Pittella, G., Kron, R.G., Koo, D.C., and Bershady, M. 1989, 'A survey for faint variable objects in SA 57', The Astronomical Journal, 98, 108-116

49. Vaughan, S., Uttley, P., Markowitz, A.G., Huppenkothen, D., Middleton, M.J., Alston, W.N., Scargle, J.D., and Farr, W.M. 2016, 'False periodicities in quasar timedomain surveys', Monthly Notices of the Royal Astronomical Society, 461, 31453152

50. Warren, S.J., Hewett, P.C., Irwin, M.J., and Osmer, P.S. 1991, 'A wide-field multicolor survey for high-redshift quasars, $z>2.2$. I - Photometric catalog and survey selection function', The Astrophysical Journal Supplement Series, 76, 1-22

51. Weinstein, M.A., Richards, G.T., Schneider, D.P., Younger, J.D., Strauss, M.A., Hall, P.B., Budavári, T., Gunn, J.E., York, D.G., and Brinkmann, J. 2004, 'An Empirical Algorithm for Broadband Photometric Redshifts of Quasars from the Sloan Digital Sky Survey', The Astrophysical Journal Supplement Series, 155, 243-256 
52. Zheng, Z.-Y., Butler, N.R., Shen, Y., Jiang, L., Wang, J.-X., Chen, X., and Cuadra, J. 2016, 'SDSS J0159+0105: A Radio-Quiet Quasar with a Centi-Parsec Supermassive Black Hole Binary Candidate', The Astrophysical Journal, 827, 56

53. Zhu, F.-F., Wang, J.-X., Cai, Z.-Y., and Sun, Y.-H. 2016, 'The Timescale-dependent Color Variability of Quasars Viewed with /GALEX', The Astrophysical Journal, 832, 75

54. Zinn, J.C., Kochanek, C.S., Kozłowski, S., Udalski, A., Szymański, M.K., Soszyński, I., Wyrzykowski, Ł., Ulaczyk, K., Poleski, R., Pietrukowicz, P., Skowron, J., Mróz, P., and Pawlak, M. 2017, 'Variable classification in the LSST era: exploring a model for quasi-periodic light curves', Monthly Notices of the Royal Astronomical Society, 468, 2189-2205 